\title{
Efeito da radiação gama nos parâmetros microbiológicos, físico-químicos e compostos fenólicos de farinha de resíduos de frutas durante armazenamento
}

Effect of gamma radiation on the microbiological and physicochemical parameters and on the phenolic compounds of a fruit residue flour during storage

\author{
Jessica Bomtorin Aranha ${ }^{1 \star}$, Talita Costa Negri', José Guilherme Prado Martin', Marta Helena Fillet Spoto \\ 1 Universidade de São Paulo (USP), Escola Superior de Agricultura "Luiz de Queiroz" (ESALQ), Departamento de Agroindústria, Alimentos e Nutrição, \\ Piracicaba/SP - Brasil
}

\section{*Corresponding Author}

Jessica Bomtorin Aranha, Universidade de São Paulo (USP), Escola Superior de Agricultura "Luiz de Queiroz" ESALQ, Departamento de Agroindústria, Alimentos e Nutrição, Av. Páduas Dias, 11 - Agronomia, CEP: 13418-900, Piracicaba/SP - Brasil, e-mail: jessica.aranha@usp.br

Cite as: Effect of gamma radiation on the microbiological and physicochemical parameters and on the phenolic compounds of a fruit residue flour during storage. Braz. J. Food Technol., v. 20, e2016123, 2017.

Received: Oct. 05, 2016; Accepted: May 03, 2017

\section{Resumo}

Resíduos agroindustriais possuem elevados teores de nutrientes, no entanto, são pouco explorados para o consumo, pois são necessários tratamentos prévios para garantir sua segurança microbiológica. A irradiação é um processo que possibilita a redução da população microbiana contaminante, bem como a manutenção das características do produto. O objetivo do trabalho foi avaliar o efeito da irradiação na qualidade microbiológica, nas propriedades físico-químicas e nos compostos fenólicos da farinha de resíduos de frutas, durante seu armazenamento. A farinha foi obtida a partir dos resíduos desidratados de abacaxi, melão, mamão e maçã e foi submetida à irradiação com doses de 0, 1, 2 e 3 kGy. Os parâmetros microbiológicos (contagem de coliformes, bolores e leveduras, e Salmonella sp.) e físico-químicos ( $\mathrm{pH}$, acidez titulável, sólidos solúveis, atividade de água, cor L, $a^{*}$ e $b^{*}$ e compostos fenólicos) foram avaliados nos tempos 0, 7, 14 e 21 dias de armazenamento. A irradiação reduziu a carga microbiana para todos os tratamentos e não foi detectada a presença de Salmonella sp. nas amostras. O pH e a acidez alteraram-se somente no armazenamento. Sólidos solúveis e atividade de água não apresentaram diferenças entre as doses durante o armazenamento. Os compostos fenólicos foram preservados, com a aplicação da irradiação. As farinhas apresentaram um leve escurecimento decorrente da aplicação da irradiação. Concluiu-se que as doses mais eficazes, com a manutenção da qualidade microbiológica e características físico-químicas da farinha, foram as doses de 2 e 3 kGy.

Palavras-chave: Subprodutos; Casca; Composição centesimal; Irradiação em alimentos.

\section{Abstract}

Agroindustrial residues have high levels of nutrients, but are little exploited for consumption because they require prior treatment to ensure microbiological safety. Irradiation is an effective process for the reduction of microbial counts with maintenance of the product characteristics. The objective of this study was to evaluate the effect of irradiation on the microbiological quality, physicochemical properties and phenolic compounds of a fruit residue flour during storage. The flour was obtained from dehydrated residues of pineapple, melon, papaya and apple, which were submitted to irradiation doses of $(0,1,2$ and $3 \mathrm{kGy})$. The microbiological (coliforms, moulds, yeast and Salmonella sp. counts) and physicochemical $\left(\mathrm{pH}\right.$, titratable acidity, soluble solids, water activity, colour parameters $\mathrm{L}^{*}, \mathrm{a}^{*}$ and $\mathrm{b}^{*}$ and phenolic compounds) parameters were evaluated after $0,7,14$ and 21 days of storage. All the irradiation treatments reduced the microbial count, and the presence of Salmonella sp was not detected in any of the samples. The acidity and pH showed changes during storage. The soluble solids and water activity showed no significant differences between the doses during storage. The phenolic compounds were preserved by the application of irradiation. The colour of the flour samples darkened slightly with irradiation. It was concluded that the most effective doses for the maintenance of the microbiological quality and physicochemical characteristics of the flour were 2 and 3 kGy.

Keywords: By-products; Peel; Proximate composition; Food irradiation. 
Efeito da radiação gama nos parâmetros microbiológicos, físico-químicos e compostos fenólicos de farinha de resíduos de frutas durante armazenamento

Aranha, J. B. et al.

\section{Introdução}

À medida que indústrias processadoras de frutas e hortaliças investem no aumento da capacidade de produção, intensificam a geração de subprodutos e resíduos agroindustriais, os quais, muitas vezes, não são reaproveitados, gerando problemas ambientais decorrentes de seu descarte (LOUSADA JUNIOR et al., 2005; SENA; NUNES, 2006). Nos últimos anos, muitos trabalhos têm avaliado seu potencial de reutilização, visando à aplicação em alimentos para animais, além de estudos voltados para a identificação de compostos e nutrientes de partes não convencionais, como o bagaço, a casca, os talos e as sementes (MATIAS et al., 2005, GONDIM et al., 2005; ROCHA et al., 2008; MARTIN et al., 2012; STORCK et al., 2015). No entanto, o reaproveitamento de resíduos ainda é pouco explorado pela indústria de alimentos.

Esses resíduos podem apresentar características nutricionais relevantes e, muitas vezes, em quantidade de fibras, minerais e compostos bioativos superiores às partes convencionais, como a polpa (MARQUES et al., 2010; SALGADO et al., 2012). Dessa maneira, poderiam ser explorados para o uso na alimentação humana, bem como para a fortificação de produtos. No entanto, para garantir sua segurança microbiológica e nutricional, faz-se necessária a aplicação de algum tipo de processamento, mecânico ou térmico (EVANGELISTA, 2005).

Nesse contexto, a irradiação de alimentos por raios gama consiste em uma técnica que possibilita a transformação de resíduos em produtos para o consumo. Essa técnica é considerada um método efetivo na redução da contaminação microbiana, garantindo a segurança microbiológica do produto, bem como o aumento de sua vida útil (MALI et al., 2011).

Em alimentos de origem vegetal, como frutas e hortaliças, o processo de irradiação tem sido utilizado visando a diferentes aplicações, como o controle de fungos causadores de podridão pós-colheita; a inativação de patógenos em frutos frescos e legumes cortados, os quais são posteriormente embalados para o comércio; no tratamento de quarentena para produtos sujeitos à infestação por pragas de insetos; na extensão da vida útil dos produtos, retardando os processos fisiológicos e bioquímicos que levam à maturação e ao amadurecimento (THOMAS, 2001). Além disso, poucos estudos avaliam o processo de irradiação em subprodutos de frutas e hortaliças. Dessa forma, há necessidade de se explorarem os efeitos da irradiação nas características dos diferentes produtos.

Diante dessa problemática, o presente trabalho teve como objetivo avaliar a eficiência da radiação gama na conservação de farinha obtida de resíduos do processamento de frutas, por meio da análise dos parâmetros físico-químicos, do teor de compostos fenólicos e da qualidade microbiológica durante o armazenamento.

\section{Material e métodos}

\subsection{Obtenção e processamento da matéria-prima}

Cerca de $46 \mathrm{~kg}$ de resíduos agroindustriais das frutas abacaxi, melão, mamão e maçã foram cedidos pela empresa Della Natural, localizada na cidade de Engenheiro Coelho - SP. Foram utilizadas a casca e as sementes de mamão e melão, as cascas de abacaxi e os talos e as sementes da maçã. Os resíduos foram lavados com água corrente e cortados em pequenos pedaços para facilitar o processo de desidratação, realizado em estufa (Marconi, MA035/3BXI/TOM) de ar forçado a $70^{\circ} \mathrm{C} \pm 2$, durante 48 horas. Após essa etapa, os resíduos secos foram triturados em moinho (IKA, A11 basic) e, posteriormente, homogeneizados manualmente para obtenção de uma farinha fina e uniforme (16 mesh). A farinha foi composta de resíduos, sendo $47,25 \%$ de abacaxi, 31,87\% de melão, $15,38 \%$ de mamão e $5,50 \%$ de maçã.

\subsection{Irradiação da farinha}

A farinha foi acondicionada em sacos plásticos de polietileno $(25 \mathrm{~cm} \times 15 \mathrm{~cm})$ em porções de $200 \mathrm{~g}$, selados (Selamaq) e transportados em temperatura ambiente $\left(25^{\circ} \mathrm{C}\right)$, em caixas isotérmicas, até o Instituto de Pesquisas Energéticas e Nucleares (IPEN), em São Paulo, onde foi submetida à irradiação em Irradiador Multipropósito Cobalto-60. Foram aplicadas doses de 0, 1, 2 e 3 kGy. A taxa de dose utilizada foi de 0,87 kGy/h, com variação menor que $1 \%$, sendo dosimetria realizada por meio do dosímetro Harwell Amber 3042. As amostras embaladas foram mantidas à temperatura ambiente em local com baixa incidência de luz e avaliadas nos tempos 0, 7, 14 e 21 dias de armazenamento.

\subsection{Composição centesimal para caracterização da matéria-prima}

Foi realizada a composição centesimal da farinha obtida de resíduos de frutas para a caracterização da matéria-prima, não sendo tal parâmetro avaliado em todos os períodos de armazenamento. Os teores de umidade, cinzas, proteínas e lipídeos foram determinados de acordo com AOAC (2005a). No caso da quantificação dos lipídeos, utilizou-se hexano como solvente extrator. O teor de fibras alimentares foi obtido de acordo com Asp et al. (1983). As amostras foram submetidas à digestão enzimática e suas frações separadas em solúveis e insolúveis. O valor calórico total foi calculado de acordo com Osborne e Voogt (1978), através dos fatores de conversão de Atwater. O teor de carboidratos foi determinado da seguinte maneira: \%Carboidratos = 100\% - \%lipídeos - \%proteínas - \%cinzas - \%fibras totais. 
Efeito da radiação gama nos parâmetros microbiológicos, físico-químicos e compostos fenólicos de farinha de resíduos de frutas durante armazenamento

Aranha, J. B. et al.

\subsection{Análises microbiológicas}

As análises microbiológicas compreenderam a pesquisa por Salmonella sp. em $25 \mathrm{~g}$, coliformes totais e termotolerantes, e a contagem de bolores e leveduras, ao longo do período de armazenamento. A pesquisa de Salmonella sp. foi realizada utilizando-se kit 1,2-Test Salmonella Rapid Test (Biocontrol Systems ${ }^{\circledR}$ ), metodologia validada pela AOAC Official Method 989.13 (AOAC, 2005b). Para pesquisa de coliformes totais e termotolerantes, foi utilizado o kit Simplate (Biocontrol Systems ${ }^{\circledR}$ ), metodologia validada pela AOAC Official Method 991.15 (AOAC, 2005c). A análise de bolores e leveduras foi realizada de acordo com Downes e Ito (2001). As análises foram realizadas para todos os períodos, exceto a análise da pesquisa de Salmonella sp., realizada somente no primeiro dia, pelas condições utilizadas no processamento e pelas características da farinha. Alguns fatores podem contribuir com esta segurança para o produto, uma vez que foram utilizadas temperaturas de desidratação em torno de $70{ }^{\circ} \mathrm{C} \pm 2{ }^{\circ} \mathrm{C}$, bem como baixo $\mathrm{pH}(<4,5)$, baixa atividade de água e higienização adequada durante todo o processamento. Estes fatores podem ser determinantes para a redução significativa da Salmonella sp. (BANWART, 1989; FORSYTHE, 2013).

\subsection{Análises físico-químicas}

Os teores de sólidos solúveis foram determinados através de leituras em refratômetro digital (Krüss Optronic - DR201-05-Alemanha), sendo os resultados expressos em ${ }^{\circ}$ Brix. A determinação do pH foi realizada em potenciômetro digital (Marcon, MA-522), de acordo com o Instituto Adolf Lutz (IAL, 1985). A acidez titulável foi determinada com solução de hidróxido de sódio $(0,1 \mathrm{~N})$, utilizando como indicador a fenolftaleína (IAL, 1985). Os parâmetros de cor L*, a* e b* foram avaliados conforme o sistema Cielab, utilizando-se colorímetro digital (Konica Minolta, CR-400). $A$ atividade de água foi detectada em analisador de atividade de água (Decagon, Aqualab 4TE).

\subsection{Compostos fenólicos}

Os compostos fenólicos foram quantificados utilizando-se o ácido gálico como padrão, sendo os resultados expressos em mg de ácido gálico por $g$ de amostra, de acordo com Singleton e Rossi (1965). Cerca de 0,5 g de amostra foi pesado e adicionado de $10 \mathrm{~mL}$ de metanol P.A. como solvente extrator, conforme metodologia de Rasheed et al. (2012), com modificações. Em seguida, foi centrifugado a $5^{\circ} \mathrm{C}$, a 6.000 rpm, durante 10 minutos. O sobrenadante foi reservado em vidro âmbar para o prosseguimento da análise. Transferiu-se a alíquota de $200 \mu \mathrm{L}$ do extrato para um tubo de ensaio e adicionaram-se 1,5 mL de água destilada e $100 \mu \mathrm{L}$ de Folin P.A. Os tubos foram agitados em vórtex e reservados por 5 minutos. Posteriormente, adicionaram-se $200 \mu \mathrm{L}$ de carbonato de sódio 20\% e agitou-se em vórtex por 30 segundos. Os tubos foram reservados no escuro por 120 minutos e lidos em espectrofotômetro (JKI UV-VIS Spectrophotometer $752 \mathrm{~N}$ ), a $765 \mathrm{~nm}$.

\subsection{Delineamento experimental}

Todas as análises da farinha de resíduos irradiada foram realizadas em triplicata. Os resultados foram submetidos à Análise da Variância (ANOVA) com delineamento em esquema fatorial $4 \times 4$, sendo 4 doses de radiação: 0, 1, 2, 3 kGy, e 4 períodos de análise: dias 0, 7, 14 e 21, sendo tais análises realizadas em triplicada, utilizando-se o software ASSISTAT 7.7 (SILVA; AZEVEDO, 2009). Posteriormente, foi aplicado o teste de Tukey $(p<0,05)$, para a comparação das médias.

\section{Resultados e discussão}

\subsection{Composição centesimal}

Os resultados referentes à composição centesimal da farinha de resíduos de frutas encontram-se na Tabela 1. O teor energético calculado (150,61 kcal/100 g) mostrou-se relativamente menor quando comparado ao de outros tipos de farinha, como as farinhas de centeio, trigo ou milho (UNICAMP, 2011), bem como ao teor de outros tipos de resíduos desidratados, como goiaba, acerola, umbu e maracujá, que apresentaram teor calórico de 266,65; 332,$53 ; 314,17$, e 254,36 kcal/100 g, respectivamente (ABUD; NARAIN, 2009). Por outro lado, a farinha obtida neste trabalho apresentou-se mais calórica que a farinha de cascas de maracujá $(85,86 \mathrm{kcal} / 100 \mathrm{~g})$, desenvolvida por Souza et al. (2008).

De acordo com a legislação brasileira destinada à composição de farinhas, permitem-se teores de umidade entre 8 e $15 \%$ no produto final (BRASIL, 1978). Dessa forma, a farinha avaliada neste estudo não estava em conformidade com a referida legislação (19,19\% de umidade). Farinhas de resíduos obtidas por outros trabalhos apresentaram teores de umidade inferiores aos observados, como 6,09\% para farinha de casca de maracujá (SOUZA et al., 2008); 5,64\%

Tabela 1. Composição centesimal da farinha de resíduos de frutas (em $100 \mathrm{~g} \mathrm{b.u.)}$.

\begin{tabular}{cc}
\hline Composição Centesimal e Valor Calórico Total \\
\hline Valor Calórico Total (kcal) & 150,61 \\
Umidade (\%) & $19,19 \pm 0,11$ \\
Proteína (\%) & $3,87 \pm 0,41$ \\
Cinza (\%) & $6,41 \pm 0,06$ \\
Lipídeos (\%) & $1,29 \pm 0,12$ \\
Fibra solúvel (\%) & $1,99 \pm 0,21$ \\
Fibra insolúvel (\%) & $36,37 \pm 0,31$ \\
Carboidratos (\%) & 30,88 \\
\hline
\end{tabular}

b. u.=base úmida. Média \pm desvio padrão. 
Efeito da radiação gama nos parâmetros microbiológicos, físico-químicos e compostos fenólicos de farinha de resíduos de frutas durante armazenamento

Aranha, J. B. et al.

para farinha de bagaço de maçã; $8,45 \%$ para farinha de bagaço de laranja; 7,38\% para farinha de cascas de maracujá (MACAGNAN et al., 2014); 8,60\% para farinha de resíduos de acerola (AQUINO et al., 2010), e 4,67\% para farinha de casca de manga (AZEVÊDO et al., 2008). Um maior período de secagem poderia ter resultado em uma farinha com menor teor de umidade; no entanto, devido à alta concentração de açúcares presentes nos resíduos empregados para sua produção, optou-se por manter a secagem durante 48 horas.

Os teores de cinzas $(6,41 \%)$ foram superiores ao obtido em estudos com farinha de resíduo de acerola, de 1,93 a 2,51\% (ABUD; NARAIN, 2009; AQUINO et al., 2010; STORCK et al., 2015); 4,16\% e 2,03\%, para farinhas e pós-alimentícios obtidos a partir de resíduos do abacaxi (COSTA, et al., 2007; MENDES, 2013), e 1,03\%, 0,82\%, $0,96 \%$, para farinha das cascas de abacaxi, mamão e melão, respectivamente (GONDIM et al., 2005). Storck et al. (2015) encontraram 4,38 a 16,86\% de matéria inorgânica nas farinhas de resíduos do processamento da maçã com diferentes granulometrias, o que está de acordo com os valores elevados de minerais encontrados no referido estudo.

A porcentagem de lipídeos mostrou-se relativamente baixa quando comparada aos demais constituintes, em média, 1,29\%. Gondim et al. (2005) observaram concentração baixas, de 0,55\%, 0,08\% e 0,10\%, para casca de abacaxi, melão e mamão, respectivamente. Costa et al. (2007) encontraram 1,60\% de lipídeos em produtos obtidos de resíduos de abacaxi; valores de 1,97 a 5,23\% foram detectados em resíduos de acerola (ABUD; NARAIN 2009; AQUINO et al., 2010) e 2,05\% para resíduos do processamento de maçã (AQUINO et al., 2010). Bertagnolli et al. (2014) caracterizaram farinha de cascas de goiaba e encontraram 1,6\% de lipídeos nesse material. Resíduos de frutos apresentam uma variação muito grande em termos de composição, a qual pode estar diretamente relacionada com as quantidades de cada um dos constituintes presentes, o que justifica as diferenças apontadas para a farinha de frutas estudada.
Em relação à porcentagem de proteínas, a farinha apresentou teores médios de 3,87\%. Mendes (2013) encontrou teor proteico de 4,49\% para a farinha da casca de abacaxi; Macagnan et al. (2014) observaram valores de 6,98, 4,89 e 5,26\%, em farinhas de bagaço de maçã, laranja e maracujá, respectivamente. Souza et al. (2008) e Aquino et al. (2010) analisaram a composição centesimal da farinha da casca do maracujá e da farinha de resíduos de acerola, e encontraram 11,76\% e 8,88\% de proteínas, respectivamente.

O produto apresentou quantidade elevadas de fibras alimentícias, pois verificou-se, em média, 39,36\% desse componente, sendo $36,37 \%$ de fibras insolúveis e 1,99\% de fibras solúveis. Segundo a Resolução RDC n. ${ }^{\circ}$ 54, de 12 de novembro de 2012 (BRASIL, 2012), do Ministério da Saúde e Agência Nacional de Vigilância Sanitária, alimentos que possuem valores mínimos de $6 \%$ de fibras podem ser classificados como de "alto conteúdo de fibras". Coelho e Wosiacki (2010) obtiveram 43,02\% de fibras totais para farinha de bagaço de maçã e Storck et al. (2015) encontraram 37,9\%, para farinhas de resíduos de acerola, quantidades tão significativas quanto às detectadas no presente estudo. Em farinha de casca de maracujá, foi encontrado 66,37\% de fibra alimentar total (SOUZA et al., 2008), e em pós-alimentícios obtidos de casca de abacaxi, apenas 7,5\% de fibras alimentares (COSTA et al., 2007).

Em relação aos carboidratos, a farinha de resíduos apresentou elevada quantidade deste componente $(30,88 \%)$, teor semelhante ao encontrado em outros estudos para farinha de bagaço de laranja $(33,12 \%)$ (MACAGNAN et al., 2014); farinha de resíduos de uva (30,5\%) (STORCK et al., 2015) e farinha de resíduos de goiaba desidratada (29,52\%) (ABUD; NARAIN, 2009).

\subsection{Análises microbiológicas}

Não foi detectada Salmonella sp. na farinha de resíduos de frutas (ausência em $25 \mathrm{~g}$ ), bem como coliformes termotolerantes ( $<1$ UFC/g). Coliformes totais foram detectados apenas na amostra controle (Figura 1).
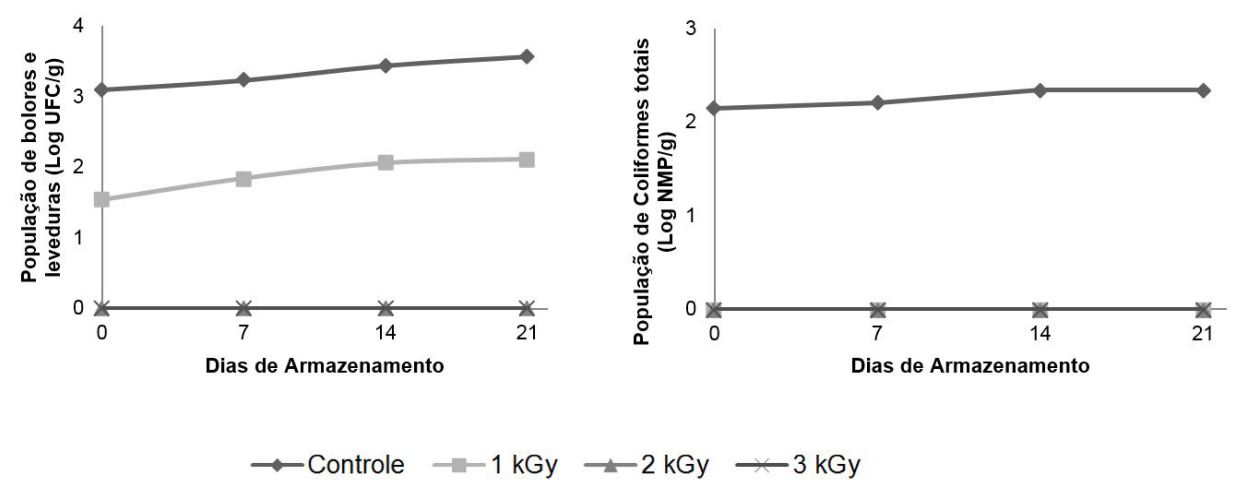

Figura 1. População de bolores e leveduras e de coliformes totais. 
Efeito da radiação gama nos parâmetros microbiológicos, físico-químicos e compostos fenólicos de farinha de resíduos de frutas durante armazenamento

Aranha, J. B. et al.

Em relação aos bolores e leveduras, foram detectadas contagens apenas nas amostras controle e nas irradiadas com 1 kGy. Desta maneira, tem-se que a dose de 1 kGy foi insuficiente para destruir os fungos e leveduras, porém reduziu à metade a contagem destes microrganismos. Jin et al. (2017) também estudaram o efeito da radiação gama (1 kGy) sobre o crescimento microbiano de raízes de ginseng e observaram que, com esta dose, foi possível somente diminuir a carga de fungos e leveduras. A irradiação com doses superiores, de 2 e $3 \mathrm{kGy}$, aplicadas no presente trabalho, contribuiu significativamente $(p<0,05)$ na redução desses contaminantes. Naresh et al. (2015) estudaram a eficácia da radiação gama (doses de 0, 0,5, 1 e 3 kGy) sobre fungos e leveduras presentes em suco de manga, responsáveis pela deterioração do produto. Como resultado, doses $\geq 0,5 \mathrm{kGy}$ foram capazes de reduzir a carga destes deteriorantes e apenas a dose de 3 kGy foi capaz de reduzir a níveis não detectáveis, assim como no presente estudo. Alighourchi et al. (2008) estudaram sucos de romã tratados com irradiação, observando que o crescimento de bactérias e fungos foi reduzido com a aplicação de 0,5 e 2 kGy; porém, apenas doses $\geq 3,5$ kGy reduziram a população microbiana a níveis não detectáveis.

Os efeitos diretos da irradiação implicam na formação de íons e radicais livres, que podem reagir com proteínas e o DNA dos microrganismos, ocasionando efeitos biológicos, como mutação de células e até mesmo a morte celular (HENRIKSEN; MAILLIE, 2002). Células danificadas pela irradiação tornaram-se inativas e, com isso, incapazes de se adaptar ao ambiente (BYUN et al., 2001). As amostras avaliadas estavam em conformidade com a legislação brasileira, podendo ser consideradas, do ponto de vista microbiológico, aptas ao consumo (BRASIL, 2001).

\subsection{Análises físico-químicas}

$\mathrm{O}$ pH das amostras apresentou uma variação de 4,39 a 4,50 (Figura 2). Para o período analisado de armazenamento, foi observado um aumento dos valores de $\mathrm{pH}$ para todos os tratamentos, sendo que não houve diferenças significativas entre as doses de irradiação aplicadas $(p<0,05)$. Segundo Mendes (2013), farinhas de casca de abacaxi e de manga apresentaram valores de $\mathrm{pH}$ muito próximos aos encontrados no presente estudo, de 4,42 e 4,54, respectivamente, demonstrando que a irradiação não afetou esse parâmetro. Estudos com farinhas de resíduo de goiaba, acerola, umbu e maracujá apresentaram pH de 3,12 a 4,88 (ABUD; NARAIN, 2009). Já em farinhas de resíduos de acerola, o pH encontrado foi 3,32, valor inferior ao observado neste estudo (AQUINO et al., 2010).

Os valores de acidez do presente trabalho variaram de 2,92 a 3,28 g ácido cítrico/100 g de amostra (Figura 2). Durante o armazenamento de 21 dias, foi observada a redução da acidez titulável para todos os tratamentos. Estes resultados estão de acordo com os valores de $\mathrm{pH}$, os quais apresentaram um aumento, para o mesmo período. Segundo Ferreira et al. (2013), a redução da acidez titulável pode estar associada às transformações dos ácidos orgânicos, entre o dióxido de carbono e a água, durante o armazenamento.
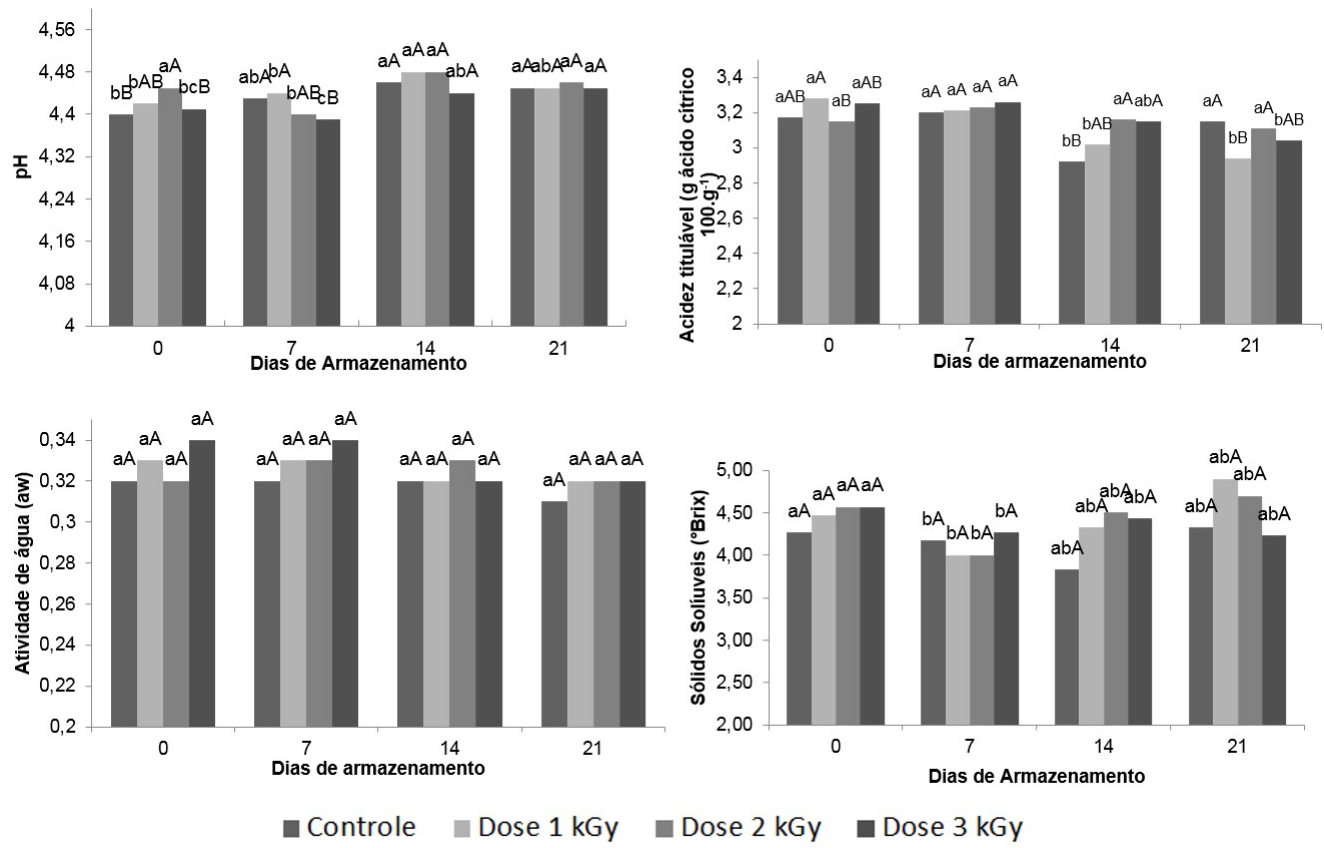

Figura 2. Análises físico-químicas da farinha de resíduos de frutas. Letras minúsculas diferentes apresentam diferença significativa das médias entre as doses $(p<0,05)$. Letras maiúsculas diferentes apresentam diferença significativa entre os dias de armazenamento $(p<0,05)$. 
Efeito da radiação gama nos parâmetros microbiológicos, físico-químicos e compostos fenólicos de farinha de resíduos de frutas durante armazenamento

Aranha, J. B. et al.

Costa et al. (2007) avaliaram produtos obtidos de casca e bagaço de abacaxi, e observaram valores de acidez variando de 2,53 a 2,98 g/100 $\mathrm{g}$. Outros autores encontraram teores inferiores de acidez titulável para farinha de casca de abacaxi $\left(1,99 \mathrm{~g} / 100 \mathrm{~g}^{1}\right)$, casca de manga $(0,19 \mathrm{~g} / 100 \mathrm{~g}$ ) (MENDES, 2013), acerola $(0,69 \mathrm{~g} / 100 \mathrm{~g})$ e resíduo de maçã (1,23 a 1,74 g/100 g) (STORCK et al., 2015). Esta variação pode ter ocorrido em função da diferença da acidez de cada matéria-prima trabalhada e do ponto de maturação dos frutos.

As amostras de farinha de resíduos de frutas analisadas apresentaram baixa atividade de água, variando de 0,31 a 0,34 (Figura 2), com média geral de 0,325 , o que já era esperado para o produto. A farinha não apresentou diferenças significativas $(p<0,05)$ entre as doses aplicadas e os dias de armazenamento avaliados. Coelho e Wosiacki (2010) analisaram a atividade de água de farinha de bagaço de maçã e encontraram teor de 0,81, superior ao obtido nesta pesquisa; além disso, nesse mesmo estudo, a farinha apresentou características microbiológicas aceitáveis pela legislação (BRASIL, 2001). Azevêdo et al. (2008) e Mendes (2013) encontraram teores de 0,4, 0,34 e 0,33, em farinha da casca de manga Tommy Atkis e farinhas de casca de abacaxi e manga, respectivamente, resultados que afirmam os dados deste estudo.

Os resultados obtidos para a concentração de sólidos solúveis ( ${ }^{\circ}$ Brix) presentes na farinha de resíduos mostraram que houve diferenças significativas $(p<0,05)$ entre os períodos de armazenamento. Os teores de sólidos solúveis no presente estudo variaram de $3,83^{\circ} \mathrm{Brix}$ a $4,57^{\circ} \mathrm{Brix}$ (Figura 2). Valores semelhantes foram determinados por Storck et al. (2015), em farinhas de resíduo de acerola e maçã, os quais obtiveram valores entre $1,07^{\circ}$ Brix e 5,10 ${ }^{\circ} \mathrm{Brix}$, corroborando com o presente estudo. Segundo os autores, a formação dos sólidos solúveis pode estar relacionada com as transformações ocorridas nas reservas durante o armazenamento, bem como com o processo de amadurecimento dos frutos (JERONIMO; KANESIRO, 2000; CHITARRA; CHITARRA, 2005).

A coloração da farinha foi avaliada de acordo com o sistema CIELab, em que o parâmetro $L^{*}$ avalia a luminosidade da amostra, representado pelo valor 100 para branco e 0 para o preto; $a^{*}$ indica coloração do vermelho $\left(+a^{*}\right)$ ao verde $\left(-a^{*}\right)$, e o parâmetro $b^{*}$ indica a coloração do amarelo $\left(+b^{\star}\right)$ ao azul $\left(-b^{*}\right)$ (PRATI et al., 2005).

Neste estudo, o parâmetro L* variou de 54,52 a 60,59 e o parâmetro $a^{*}$ apresentou valores que variaram de 3,29 a 5,29 (Figura 3). A irradiação reduziu os valores de $L^{*}$, diferindo significativamente do controle $(p<0,05)$. Já para o parâmetro $a^{*}$, houve aumento dos valores durante o armazenamento. Com isso, tem-se que tanto a irradiação quanto o armazenamento podem favorecer alterações da coloração da farinha, a qual apresentou escurecimento. O mesmo foi relatado por Alves et al. (2014), em farinhas de jabuticaba, e por Kortei et al. (2015), em cogumelos irradiados. Este último trabalho observou que a interação entre a embalagem e a irradiação causou significativa alteração nas cores durante o armazenamento. A variação observada para o parâmetro b* foi de 29,15 a 31,18, sendo que os tratamentos não apresentaram diferenças significativas durante 0 armazenamento, como também entre as doses de irradiação aplicadas (Figura 3).

Este fato pode ser explicado, uma vez que a coloração dos produtos é uma característica importante, pois influencia as escolhas dos consumidores. Além disso, durante o processamento e o armazenamento, podem ocorrer mudanças na coloração dos produtos, induzidas pela reação de Maillard, por escurecimento enzimático,
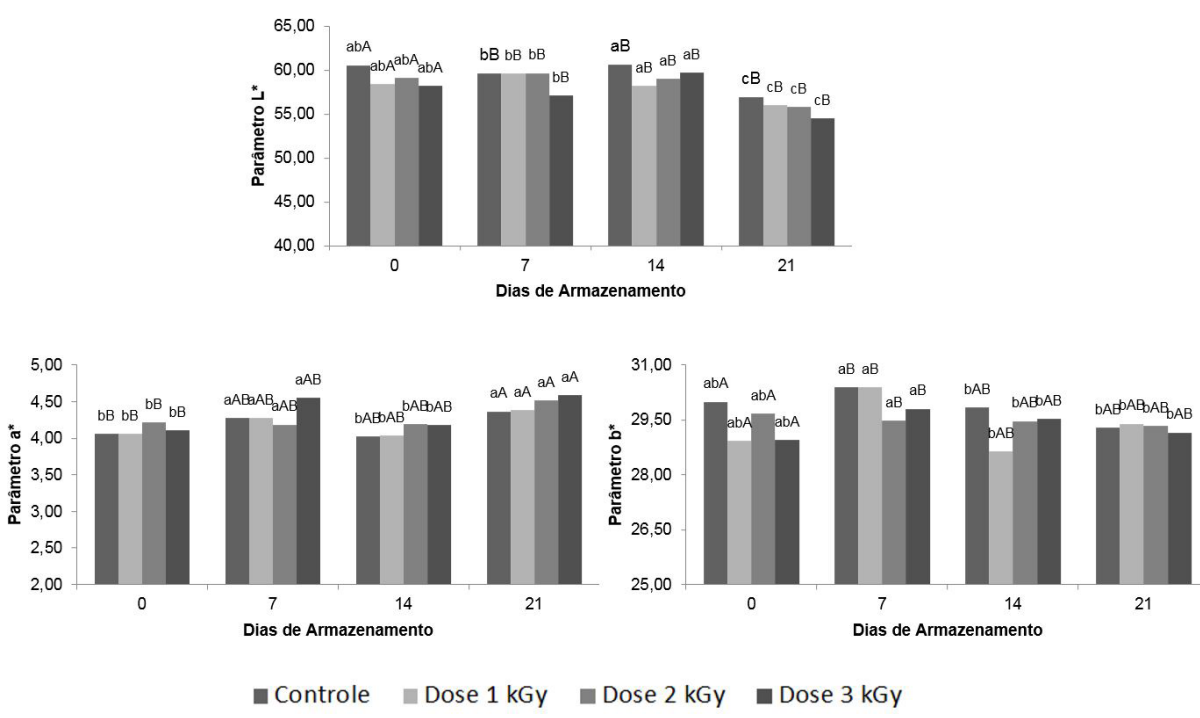

Figura 3. Parâmetros de cor $L^{*}, a^{*}$ e $b^{*}$. Letras minúsculas diferentes apresentam diferença significativa das médias entre as doses $(p<0,05)$. Letras maiúsculas diferentes apresentam diferença significativa entre os dias de armazenamento $(p<0,05)$. 


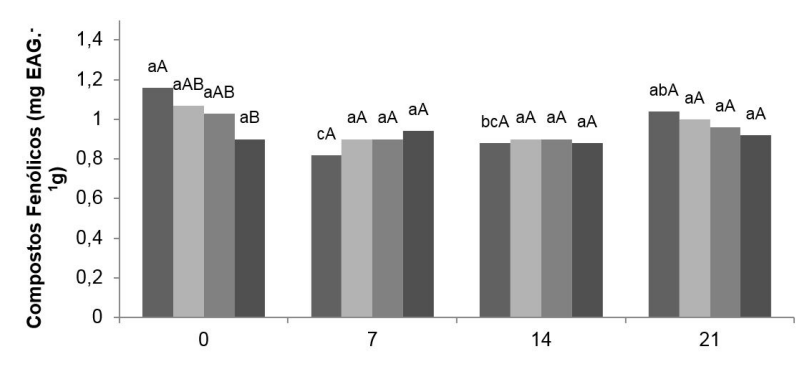

- Controle Dose 1 kGy Dose 2 kGy Dose 3 kGy

Figura 4. Compostos fenólicos da farinha de resíduos de frutas. Letras minúsculas diferentes apresentam diferença significativa das médias entre as doses $(p<0,05)$. Letras maiúsculas diferentes apresentam diferença significativa entre os dias de armazenamento $(p<0,05)$.

alterações no pH, na acidez e na temperatura de armazenamento, e por degradação de pigmentos, o que pode ser uma consequência do processo de irradiação (SHAHBAZ et al., 2014; NARESH et al., 2015), justificando os possíveis motivos para a alteração da coloração da farinha avaliada.

De acordo com alguns autores, os compostos fenólicos atuam como sequestradores de radicais livres, prevenindo o processo de autoxidação, através de mecanismos, como, por exemplo, a complexação de metais e a inativação de oxigênio singlete; por essa característica, são capazes de reduzir o risco de doenças, como câncer e aterosclerose, quando adicionados, consumidos na alimentação humana (NAMIKI, 1990; SHAHIDI et al., 1992; RAMARATHNAM et al., 1995; ADEGOKE et al., 1998; ANGELO; JORGE, 2007).

Os teores de compostos fenólicos apresentaram valores de 0,82 a 1,16 mg de ácido gálico por grama de amostra (Figura 4), demonstrando que, mesmo com o processamento térmico da secagem da farinha, a mesma apresentou teores de compostos fenólicos elevados, em comparação a outros estudos com farinhas (SOUZA et al., 2011; STORCK et al., 2015). O controle apresentou queda no teor destes compostos nos dois primeiros períodos de armazenamento, o que demonstra que houve diferenças significativas quanto ao tempo de armazenamento para os mesmos períodos $(p<0,05)$. Esta variação do teor dos compostos fenólicos pode ser observada no controle pela oscilação durante o armazenamento, uma vez que as amostras irradiadas não apresentaram alterações significativas $(p<0,05)$ no período analisado, resultado que se mostra interessante devido à importância desses compostos nos alimentos. Dessa forma, as amostras expostas à irradiação apresentaram maior estabilidade no decorrer do armazenamento.

Mali et al. (2011) avaliaram o efeito da irradiação em produtos obtidos de cascas de romã e observaram que as doses de irradiação de 5, 15 e 25 kGy não afetaram a concentração de compostos fenólicos, bem como não houve alterações significativas após um mês de armazenamento. No entanto, no mesmo estudo, a dose de 10 kGy proporcionou um aumento destes compostos, após 30 dias de armazenamento, assim como observado no presente estudo.

Storck et al. (2015) estudaram a composição de farinhas e o teor de polifenóis de resíduos da produção de suco de algumas frutas, entre as quais a laranja, a acerola, o maracujá e a uva, e observaram elevada presença de polifenóis nas farinhas, variando de 0,222 a $3,81 \mathrm{mg} / \mathrm{g}$ de farinha. Bertagnolli et al. (2014) avaliaram o teor de compostos fenólicos de farinha de casca de goiaba e observaram que, após o processamento para a obtenção de cookies, o teor destes compostos diminuiu. Diante disso, podem-se justificar as pequenas variações do teor dos compostos fenólicos do presente estudo pelo processamento que as cascas passaram até a obtenção da farinha e pelo período de armazenamento.

\section{Conclusão}

A irradiação nas doses de 2 e 3 kGy mostraram-se eficazes na eliminação de microrganismos, sem provocar mudanças significativas nos parâmetros avaliados. Dessa forma, pode ser viabilizado o uso da farinha de resíduos de frutas irradiada como ingrediente alimentício, contribuindo para a redução de resíduos provenientes das indústrias processadoras.

\section{Agradecimentos}

À empresa Della Natural, pelo fornecimento dos resíduos agroindustriais. Ao IPEN, pela aplicação da irradiação das amostras. Ao Grupo de Extensão em Frutas e Hortaliças (GEFH/ESALQ/USP). À Prof. ${ }^{a}$ Dr. ${ }^{a}$ Sonia M. de Stefano Piedade, pelo auxílio no delineamento experimental.

\section{Referências}

ABUD, A. K. S.; NARAIN, N. Incorporação da farinha de resíduo do processamento de polpa de fruta em biscoitos: uma alternativa de combate ao desperdício. Brazilian Journal of Food Technology, v. 12, n. 4, p. 257-265, 2009. http://dx.doi. org/10.4260/BJFT2009800900020.

ADEGOKE, G. O.; VIJAY KUMAR, M.; GOLAPA KRISHNA, A. G.; VARADARAJ, M. C.; SAMBAIAH, K.; LOKESH, B. R. Antioxidants and lipid oxidation in food: a critical appraisal. Journal of Food Science and Technology, v. 35, n. 4, p. 283-398, 1998.

ALIGHOURCHI, H.; BARZEGAR, M.; ABBASI, S. Effect of gamma irradiation on the stability of anthocyanins and shelf-life of various pomegranate juices. Food Chemistry, v. 110, n. 4, p. 1036-1040, 2008. PMid:26047299. http://dx.doi.org/10.1016/j. foodchem.2008.03.013. 
Efeito da radiação gama nos parâmetros microbiológicos, físico-químicos e compostos fenólicos de farinha de resíduos de frutas durante armazenamento

Aranha, J. B. et al.

ALVES, A. P. C.; CORRÊA, A. D.; LINO, J. B. R.; MARQUES, T.

R. Physicochemical properties of jaboticaba skin flour stored at room temperature. Boletim do Centro de Pesquisa de Processamento de Alimentos, v. 32, n. 2, p. 271-280, 2014.

ANGELO, P. M.; JORGE, N. Phenolic compounds in foods: a brief review. Revista Instituto Adolfo Lutz, v. 66, n. 1, p. 232-240, 2007

AQUINO, A. C. M. S.; MÓES, R. S.; LEÃO, K. M. M.; FIGUEIREDO, A. V. D.; CASTRO, A. A. Avaliação físico-química e aceitação sensorial de biscoitos tipo cookies elaborados com farinha de resíduos de acerola. Revista do Instituto Adolfo Lutz, v. 69, n. 3, p. 379-386, 2010.

ASP, N. G.; JOHANSSON, C. G.; HALLMER, H.; SILJESTROEM, M. Rapid enzmatic assay for insoluble and soluble dietary fibre 1. Agriculture and Food Chemistry, v. 31, n. 3, p. 476-482, 1983. PMid:6309935. http://dx.doi.org/10.1021/jf00117a003.

ASSOCIATION OF OFFICIAL ANALYTICAL CHEMISTS - AOAC. Official methods of analysis of the Association Analytical Chemists. 18th ed. Gaithersburg: AOAC, 2005a. 1298 p.

ASSOCIATION OF OFFICIAL ANALYTICAL CHEMISTS - AOAC. Official methods of analysis of the Association Analytical Chemists. Gaithersburg: AOAC, 2005b. Disponível em: <http:// www.aoac.org /testkits/kits-microbiology.htm>. Acesso em: 15 jun. 2016.

ASSOCIATION OF OFFICIAL ANALYTICAL CHEMISTS - AOAC. Official methods of analysis of the Association Analytical Chemists. Gaithersburg: AOAC, 2005c. Disponível em: <http:// www.aoac.org/vmeth/newsmtd. htm>. Acesso em: 16 jun. 2016.

AZEVÊDO, L. C.; AZOUBEL, P. M.; SILVA, I. R. A.; ARAÚJO, A. J. B.; OLIVEIRA, S. B. Caracterização físico-química de farinha de casca de manga vc. Tommy Atkins. In: CONGRESSO BRASILEIRO DE CIÊNCIA E TECNOLOGIA DE ALIMENTOS, 21.; SEMINÁRIO LATINO AMERICANO E DO CARIBE DE CIÊNCIA E TECNOLOGIA DE ALIMENTOS, 15., 2008, Belo Horizonte. Anais... Campinas: SBCTA, 2008. Disponível em: <http://ainfo. cnptia.embrapa.br/digital/bitstream/CPATSA-2009-09/39508/1/ OPB1989.pdf>. Acesso em: 10 jun. 2016.

BANWART, G. J. Basic food microbiology. 2nd. ed. New York: Van Nostrand Rheinhold, 1989.

BERTAGNOLLI, S. M. M.; SILVEIRA, M. L. R.; FOGAÇA, A. O.; UMANN, L.; PENNA, N. G. Bioactive compounds and acceptance of cookies made with guava peel flour. Food Science and Technology, v. 34, n. 2, p. 303-308, 2014.

BRASIL. Ministério da Saúde. Agência Nacional de Vigilância Sanitária. Resolução - CNNPA n 12, de 1978. Normas técnicas especiais do Estado de São Paulo, revistas pela CNNPA, relativas a alimentos (e bebidas), para efeito em todo território brasileiro. Diário Oficial [da] República Federativa do Brasil, Brasília, DF, 24 jul. 1978.
BRASIL. Ministério da Saúde. Agência Nacional de Vigilância Sanitária. Resolução $n^{\circ} 12$, de 2 de janeiro de 2001. Regulamento técnico sobre os padrões microbiológicos para alimentos. Diário Oficial [da] República Federativa do Brasil, Brasília, DF, 12 jan. 2001

BRASIL. Ministério da Saúde. Agência Nacional de Vigilância Sanitária. Resolução RDC n 54, de 12 de novembro de 2012. Dispõe sobre o Regulamento Técnico sobre informação nutricional complementar. Diário Oficial [da] República Federativa do Brasil, Brasília, DF, 13 nov. 2012.

BYUN, M. W.; KIM, D. H.; YOOK, H. S.; KIM, J. O. Alterações nas qualidades microbiológicas e gerais em Doenjang (pasta de soja fermentada). Food Science and Biotechnology, v. 10, p. 7-11, 2001.

CHITARRA, M. I. F.; CHITARRA, A. B. Pós-colheita de frutas e hortaliças: fisiologia e manejo. 2. ed. Lavras: UFLA, 2005. 783 p.

COELHO, L. M.; WOSIACKI, G. Avaliação sensorial de produtos panificados com adição de farinha de bagaço de maçã. Revista Ciências e Tecnologia de Alimentos, v. 30, n. 3, p. 582-588, 2010.

COSTA, J. M. C.; FELIPE, E. M. F.; MAIA, G. A.; BRASIL, I. M.; HERNANDEZ, F. F. H. Comparação dos parâmetros físico-químicos e químicos de pós alimentícios obtidos de resíduos de abacaxi. Revista Ciência Agronômica, v. 38, n. 2, p. 228-232, 2007.

DOWNES, F. P.; ITO, K. Compendium of methods for the microbiological examination of foods. 4th ed. Washington: American Public Health Association, 2001. 676 p.

EVANGELISTA, J. Tecnologia de alimentos. 2. ed. São Paulo: Atheneu, 2005. 652 p.

FERREIRA, M. S. L.; SANTOS, M. C. P.; MORO, T. M. A.; BASTO, G. J.; ANDRADE, R. M. S.; GONÇALVES, É. C. B. A. Formulation and characterization of functional foods based on fruit and vegetable residue flour. Journal of Food Science and Technology, v. 52, n. 2, p. 822-830, 2013. http://dx.doi. org/10.1007/s13197-013-1061-4.

FORSYTHE, S. J. Microbiologia de segurança dos alimentos. 2. ed. Porto Alegre: Artmed, 2013. 607 p.

GONDIM, J. A. M.; MOURA, M. F. V.; DANTAS, A. S.; MEDEIROS, R. L. S.; SANTOS, K. M. Composição centesimal e de minerais em cascas de frutas. Ciências e Tecnologia de Alimentos, v. 24, n. 4, p. 825-827, 2005.

HENRIKSEN, T.; MAILLIE, H. D. Radiation \& health. New York: Taylor \& Francis, 2002. $240 \mathrm{p}$

INSTITUTO ADOLFO LUTZ - IAL. Normas analíticas do Instituto Adolfo Lutz: métodos químicos e físicos para análises de alimentos. 3. ed. São Paulo, 1985. v. 1, 533 p.

JERONIMO, R. F.; KANESIRO, M. A. B. Efeito da associação de armazenamento sob refrigeração e atmosfera modificada na qualidade de mangas 'Palmer'. Revista Brasileira de Fruticultura, v. 22, n. 2, p. 237-243, 2000. 
Efeito da radiação gama nos parâmetros microbiológicos, físico-químicos e compostos fenólicos de farinha de resíduos de frutas durante armazenamento

Aranha, J. B. et al.

JIN, T. Z.; HUANG, M.; NIEMIRA, B. A.; CHENG, L. Microbial reduction and sensory quality preservation of fresh ginseng roots using nonthermal processing and antimicrobial packaging. Food Processing and Preservation, v. 41, n. 1, p. 1-10, 2017. http:// dx.doi.org/10.1111/jfpp. 12871 .

KORTEI, N. K.; ODAMTTEN, G. T.; OBODAI, M.; APPIAH, V.; AKONOR, P. T. Determination of color parameters of gamma irradiated fresh and dried mushrooms during storage. Croatian Journal of Food Technology, Biotechnology and Nutrition, v. 10, n. 1-2, p. 66-71, 2015.

LOUSADA JUNIOR, J. E. L.; NEIVA, J. N. M.; RODRIGUEZ, N. M.; PIMENTEL, J. C. M.; LÔBO, R. N. B. Consumo e digestibilidade de subprodutos do processamento de frutas em ovinos. Revista Brasileira de Zootecnia, v. 34, n. 2, 2005. http://dx.doi.org/10.1590/ S1516-35982005000200036.

MACAGNAN, F. T.; MOURA, F. R. A.; SANTOS, L. R.; BIZZANI, M.; SILVA, L. P. Caracterização nutricional e resposta sensorial de pães de mel com alto teor de fibra alimentar elaborados com farinhas de subprodutos do processamento de frutas. Boletim do Centro de Pesquisa de Processamento de Alimentos, v. 32, n. 2, p. 201-210, 2014

MALI, A. B.; KHEDKAR, K.; LELE, S. S. Effect of gamma irradiation on total phenolic content and in vitro antioxidant activity of pomegranate (Punica granatum L.) Peels. Food and Nutrition Sciences, v. 2, n. 5, p. 428-433, 2011. http://dx.doi.org/10.4236/ fns.2011.25060.

MARQUES, A.; CHICAYBAM, G.; ARAUJO, M. T.; MANHÃES, L. R. T.; SABAA-SRUR, A. U. O. Mango rind and pulp (Mangifera indica L.) cv. Tommy Atkins centesimal composition and minerals contents. Revista Brasileira de Fruticultura, v. 32, n. 4, p. 1206-1210, 2010.

MARTIN, J. G. P.; PORTO, E.; CORRÊA, C. B.; ALENCAR, S. M.; GLORIA, E. M.; CABRAL, I. S. R.; AQUINO, L. M. Antimicrobial potential and chemical composition of agro-industrial wastes. Journal of Natural Products, v. 5, p. 27-36, 2012.

MATIAS, M. F. O.; OLIVEIRA, E. L.; GERTRUDES, E.; MAGALHAES, M. M. A. Use of fibres obtained from the cashew (Anacardium ocidentale L.) and guava (Psidium guayava) fruits for enrichment of food products. Brazilian Archives of Biology and Technology, v. 48, p. 143-150, 2005.

MENDES, B. A. Obtenção, caracterização e aplicação de farinhas das cascas de abacaxi e manga. 2013. 81 f. Dissertação (Mestrado de Engenharia de Alimentos)-Universidade Estadual do Sudoeste da Bahia, Itapetininga, 2013.

NAMIKI, M. Antioxidants/antimutagens in food. Critical Reviews in Food Science and Nutrition, v. 29, n. 4, p. 273-300, 1990. PMid:2257080. http://dx.doi.org/10.1080/10408399009527528.

NARESH, K.; VARAKUMAR, S.; VARIVAR, P. S.; SHARMA, A.; REDDY, O. V. S. Effect of -irradiation on physico-chemical and microbiological properties of mango (Mangifera indica L.) juice from eight Indian cultivars. Biosciência Alimentar, v. 12, p. 1-9, 2015.

OSBORNE, D. R.; VOOGT, P. The analysis in nutrient of foods London: Academic, 1978. 158 p.

PRATI, P.; MORETTI, R. H.; CARDELLO, H. M. A. B. Elaboration of beverage composed by blends of clarified-stabilized sugar cane juice and juice's acid fruits. Ciência e Tecnologia de Alimentos, v. 25, n. 1, p. 147-152, 2005.

RAMARATHNAM, N.; OSAWA, T.; OCHI, H.; KAWAKISHI, S. The contribution of plant food antioxidants to humans health. Trends Food Science Nutrition, v. 6, n. 3, p. 75-82, 1995. http://dx. doi. org/10.1016/S0924-2244(00)88967-0.

RASHEED, A. A.; COBHAM, E. I.; ZEIGHMAMI, M.; ONG, S. P. Extraction of phenolic compounds from pineapple fruit. In: INTERNATIONAL SYMPOSIUM ON PROCESSING \& DRYING OF FOODS, FRUITS \& VEGETABLES, 2., 2012. Malaysia. Proceedings... Malaysia: University of Nottingham, 2012. Disponível em: <https:// www.researchgate.net/publication/306033959_EXTRACTION_ OF_PHENOLIC_COMPOUNDS_FROM_PINEAPPLE_FRUIT>. Acesso em: 15 jun. 2016.

ROCHA, S. A.; LIMA; G. P. P.; LOPES, A. M.; BORGUINI, M. G.; CICCONE, V. R.; BELUTA, I. Fibras e lipídios em alimentos vegetais oriundos do cultivo orgânico e convencional. Revista Simbio-Logias, v. 1, n. 2, p. 1-9, 2008.

SALGADO, J. M.; FERREIRA, T. R. B.; BIAZOTTO, F. O.; DIAS, C. T. S. Increased antioxidant content in juice enriched with dried extract of pomegranate (Punica granatum) peel. Plant Foods for Human Nutrition, v. 67, n. 1, p. 39-43, 2012. PMid:22392496. http://dx.doi.org/10.1007/s11130-011-0264-y.

SENA, R. F.; NUNES, M. L. Utilização de resíduos agroindustriais no processamento de rações para carcinicultura. Revista Brasileira de Saúde e Produção Animal, v. 7, n. 2, p. 94-102, 2006.

SHAHBAZ, H. M.; AHN, J. J.; AKRAM, K.; KIM, H. Y.; PARK, E. J.; KWON, J. H. Chemical and sensory quality of fresh pomegranate fruits exposed to gamma radiation as quarantine treatment. Food Chemistry, v. 145, p. 312-318, 2014. PMid:24128483. http:// dx.doi.org/10.1016/j.foodchem.2013.08.052.

SHAHIDI, F.; JANITHA, V. K.; WANASUNDARA, P. D. Phenolic antioxidants. Critical Reviews in Food Science and Nutrition, v. 32, n. 1, p. 67-103, 1992. PMid:1290586. http://dx.doi. org/10.1080/10408399209527581.

SILVA, F. A. S.; AZEVEDO, C. A. V. Principal components analysis in the software assistat-statistical attendance. In: WORLD CONGRESS ON COMPUTERS IN AGRICULTURE, 7., 2009, Reno. Proceedings... Reno: American Society of Agricultural and Biological Engineers, 2009. Disponível em: <http://www. assistat.com/indexp.html>. Acesso em: 18 jun. 2016.

SINGLETON, V. L.; ROSSI, J. A. Colorimetry of total phenolics with phosphomolybdic-phosphotungstic acid reagents. American Journal of Enology and Viticulture, v. 16, n. 3, p. 144-158, 1965. 
Efeito da radiação gama nos parâmetros microbiológicos, físico-químicos e compostos fenólicos de farinha de resíduos de frutas durante armazenamento

Aranha, J. B. et al.

SOUZA, M. S. B.; VIEIRA, L. M.; SILVA, M. J. M.; LIMA, A. Caracterização nutricional e compostos antioxidantes em resíduos de polpas de frutas tropicais. Ciência Agrotecnológica, v. 35, n. 3, p. 554-559, 2011

SOUZA, M. W. S.; FERREIRA, T. B. O.; VIEIRA, I. F. R. Composição centesimal e propriedades funcionais tecnológicas da farinha da casca do maracujá. Alimentos e Nutrição, v. 19, n. 1, p. 33-36, 2008.

STORCK, C. R.; BASSO, C.; FAVARIN, F. R.; RODRIGUES, A. C. Qualidade microbiológica e composição de farinhas de resíduos da produção de suco de frutas em diferentes granulometrias. Brazilian Journal of Food Technolology, v. 18, n. 4, p. 277-284, 2015. http://dx.doi.org/10.1590/1981-6723.1615.

THOMAS, P. Irradiation of fruits and vegetables. In: MOLINS, R. A. Food irradiation: principles and applications. New York: Jonh Wiley \& Sons, 2001. cap. 8, p. 213-240.

UNIVERSIDADE ESTADUAL DE CAMPINAS - UNICAMP. Tabela brasileira de composição de alimentos -TACO. 4. ed Campinas: UNICAMP; NEPA, 2011. 161 p. 